\title{
THE PROBLEM OF MAYER WITH VARIABLE END POINTS*
}

\author{
BY \\ GILBERT AMES BLISS
}

Let $y$ denote a set of variables $\left(y_{1}, \cdots, y_{n}\right)$ each of which is a function of $x$, and let $y\left(x_{1}\right), y\left(x_{2}\right)$ be symbols for the end values of these functions at $x_{1}$ and $x_{2}$. The latter will also be represented when convenient by the sets $\left(y_{11}, \cdots, y_{n 1}\right),\left(y_{12}, \cdots, y_{n 2}\right)$, respectively. The problem to be considered is the determination of the properties of an arc

$$
y=\bar{y}(x) \quad\left(x_{1} \leqq x \leqq x_{2}\right)
$$

which satisfies a system of differential equations

$$
\varphi_{\mu}\left(x, y, y^{\prime}\right)=0 \quad(\mu=1,2, \cdots, m<n)
$$

and minimizes the first of a set of functions

$$
f_{\rho}\left(x_{1}, y\left(x_{1}\right), x_{2}, y\left(x_{2}\right)\right) \quad(\rho=1,2, \cdots, r \leqq 2 n+2)
$$

while the rest $f_{2}, \cdots, f_{n}$ are made to vanish. It will be shown that if $\Omega$ denotes the function

$$
\Omega=\lambda_{1} \phi_{1}+\cdots+\lambda_{m} \phi_{m},
$$

then a necessary condition for a minimum is that there shall exist $m$ functions $\lambda_{1}(x), \cdots, \lambda_{m}(x)$ not all identically zero on $x_{1} x_{2}$, satisfying the equations $\dagger$

$$
\Omega_{y_{k}}-\frac{d}{d x} \Omega_{y_{k^{\prime}}}=0 \quad(k=1,2, \cdots, n)
$$

and making all determinants of order $r+1$ of the matrix

$$
\left\|\begin{array}{cccc}
\frac{\partial f_{\rho}}{\partial x_{1}} & \frac{\partial f_{\rho}}{\partial y_{k 1}} & \frac{\partial f_{\rho}}{\partial x_{2}} & \frac{\partial f_{\rho}}{\partial y_{k 2}} \\
\Omega\left(x_{1}\right)-\sum_{k=1}^{n} \Omega_{y_{k^{\prime}}}\left(x_{1}\right) y_{k 1}^{\prime} & \Omega_{y_{k^{\prime}}}\left(x_{1}\right) & -\Omega\left(x_{2}\right)+\sum_{k=1}^{n} \Omega_{y_{k^{\prime}}}\left(x_{2}\right) y_{k 2}^{\prime} & -\Omega_{y_{k^{\prime}}}\left(x_{2}\right)
\end{array}\right\|
$$

vanish. In the rows of the matrix, $k=1, \cdots, n$, while in the columns

* Presented to the Society December 22, 1916, with the title, $A$ device applicable to the problems of Lagrange and Mayer in the calculus of variations.

$\dagger$ The subscripts denote partial derivatives of $\Omega$. 
$\rho=1, \cdots, r$, and the arguments in the derivatives of the functions $f_{\rho}$, and in $\Omega$ and its derivatives, are in every case those belonging to $E$.

The classical problem of Mayer in the calculus of variations is a special case of this when $f_{1}=y_{12}$ and $f_{2}, \cdots, f_{r}$ are the functions

$$
\begin{aligned}
& x_{1}-\alpha_{1}, \quad y_{j 1}-\beta_{j 1}, \quad x_{2}-\alpha_{2}, \quad y_{k 2}-\beta_{k 2} \\
& (j=1, \cdots, n ; k=2, \cdots, n),
\end{aligned}
$$

the $\alpha$ 's and $\beta$ 's being constants. In a recent paper Bolza* has formulated a problem in which he admits so-called finite equations not involving derivatives $y^{\prime}$ in the system (1), and for which the expression to be minimized, in the non-parametric case, is a sum

$$
U=\int_{x_{1}}^{x_{2}} f\left(x, y, y^{\prime}\right) d x+G\left(x_{1}, y\left(x_{1}\right), x_{2}, y\left(x_{2}\right)\right)
$$

instead of the function $f_{1}$. When $G=0$ this is the Lagrange form of problem, and when $f=0$ it has the form stated in the first paragraph above.

It will be shown in a later section that the problem of Mayer with variable end points, stated in the first paragraph above, and the problem of Bolza are equivalent in the sense that the Lagrange multiplier rule for each is deducible from that of the other. Each of them contains all of the classical problems of the calculus of variations not involving partial derivatives as special cases, with the exception of those of a type exemplified by an example proposed by Hadamard. $\dagger$ But the results for the problem which is the principal study of this paper are the most symmetrical. The proofs given below are new, an important feature being the use and symmetric treatment of the hypothesis that the matrix $\left\|\partial \varphi_{\mu} / \partial y_{k}^{\prime}\right\|(\mu=1, \cdots, m ; k=1, \cdots, n)$ is of rank $m$ at every point of $E$, instead of the usual unsymmetric assumption that a particular one of the determinants of order $m$ of the matrix is everywhere different from zero on $E$.

\section{Preliminary notions and theorems}

In the following pages it will be understood that the various indices unless otherwise expressly stated have the ranges

$i, j, k=1, \cdots, n ; \mu, \nu=1, \cdots, m ; \rho, \sigma=1, \cdots, r ; \tau=m+1, \cdots, n$.

An equation written with one of these indices is supposed to hold for every one of its values.

* Üeber den " anormalen Fall" beim Lagrangeschen und Mayerschen Problem mit gemischten Bedingungen und variabeln Endpunkten, $\mathrm{M}$ a the $\mathrm{m}$ a t i s $\mathrm{ch}$ e A n ale $\mathrm{n}$, vol. 74 (1913), p. 403.

$\dagger$ Leçons sur le calcul des variations, p. 176. 
The arc $E$ is supposed to have the following properties:

1. It is of class $C^{\prime \prime *}$ and such that the functions $\varphi_{\mu}, f_{\rho}$ are also of class $C^{\prime \prime}$ near the values of their arguments on $E$.

2. It satisfies the equations $\varphi_{\mu}=f_{2}=\cdots=f_{n}=0$.

3. The matrix $\left\|\partial \varphi_{\mu} / \partial y_{k}^{\prime}\right\|$ has rank $m$ at every point of $E$.

4. The matrix

$$
\left\|\begin{array}{llll}
\frac{\partial f_{p}}{\partial x_{1}} & \frac{\partial f_{p}}{\partial y_{k 1}} & \frac{\partial f_{p}}{\partial x_{2}} & \frac{\partial f_{p}}{\partial y_{k 2}}
\end{array}\right\|
$$

with $2 n+2$ columns and $r$ rows is of rank $r$ at the values of the arguments of the functions $f_{\rho}$ on $E$.

The last two hypotheses are to secure the independence of the differential equations and of the functions $f_{\rho}$ near $E$.

It will be shown in Section 3 , as a consequence of hypothesis 3 , that $n-m$ functions $\varphi_{\tau}\left(x, y, y^{\prime}\right)$ can always be adjoined to the $m$ functions $\varphi_{\mu}$ in such a way that the complete set $\varphi_{i}$ has the properties of Hypothesis 1 and a determinant $\left|\partial \varphi_{i} / \partial y_{k}^{\prime}\right|$ different from zero everywhere on $E$. If $z$ represents always a set $\left(z_{1}, \cdots, z_{n}\right)$ of which the first $m$ elements are identically zero, then the arc $E$ determines uniquely the $n-m$ remaining functions in $z$ so that

$$
\varphi_{k}\left(x, y, y^{\prime}\right)-z_{k}=0 \text {. }
$$

This is the extended system of differential equations formed from the system (1).

Consider a one-parameter family of arcs

$$
y_{k}=y_{k}(x, u), \quad z_{k}=z_{k}(x, u) \quad\left[x_{1}(u) \leqq x \leqq x_{2}(u)\right]
$$

which contain $E$ for $u=0$ and satisfy $f_{2}=\cdots=f_{r}=0$ and the extended system of differential equations for every $u$. Its variations are the expressions

(4) $\xi_{1}=x_{1 u}(0), \xi_{2}=x_{2 u}(0), \eta_{k}(x)=y_{k u}(x, 0), \zeta_{k}(x)=z_{k u}(x, 0)$, where the subscript $u$ denotes differentiation. It is understood that $\zeta$ as well as $z$ always represents a set of elements $\left(\zeta_{1}, \cdots, \zeta_{n}\right)$ in which the first $m$ are zero. The variations satisfy the equations of variation

where

$$
\Phi_{i}(\eta)-\zeta_{i}=F_{2}(\xi, \eta)=\cdots=F_{r}(\xi, \eta)=0
$$

$$
\begin{gathered}
\Phi_{i}(\eta)=\sum_{k}\left(\frac{\partial \varphi_{i}}{\partial y_{k}} \eta_{k}+\frac{\partial \varphi_{i}}{\partial y_{k}^{\prime}} \eta_{k}^{\prime}\right) \\
F_{\rho}(\xi, \eta)=\frac{\partial f_{\rho}}{\partial x_{1}} \xi_{1}+\sum_{k} \frac{\partial f_{\rho}}{\partial y_{k 1}}\left(y_{k 1}^{\prime} \xi_{1}+\eta_{k 1}\right)+\frac{\partial f_{\rho}}{\partial x_{2}} \xi_{2} \\
+\sum_{k} \frac{\partial f_{\rho}}{\partial y_{k 2}}\left(y_{k 2}^{\prime} \xi_{2}+\eta_{k 2}\right),
\end{gathered}
$$

*A function is of class $C^{\prime \prime}$ if it has continuous first and second derivatives. 
and the functions $y, y^{\prime}$ occurring explicitly and in the derivatives of $\varphi_{i}$ and $f_{\rho}$ are those defining $E$.

The existence theorems for linear differential equations justify the following lemma:

LEMma 1. The equations

$$
\Phi_{i}(\eta)-\zeta_{i}=0
$$

determine uniquely a set of solutions $\eta_{i}$ of class $C^{\prime \prime}$ in a neighborhood of the values $x_{1} \leqq x \leqq x_{2}$, when functions $\zeta_{i}$ of class $C^{\prime}$ in this neighborhood and the initial values of the $\eta$ 's at a single point are given.

Consider now a system $H$ of $r$ sets of variations $\xi_{1}^{\sigma}, \xi_{2}^{\sigma}, \eta_{k}^{\sigma}, \zeta_{k}^{\sigma}$ with $\zeta$ 's of class $C^{\prime}, \eta$ 's of class $C^{\prime \prime}$, and satisfying the equations (6). Variations of this sort with $\xi_{1}, \xi_{2}$ arbitrary constants are called admissible variations. Then with the help of the existence theorems for implicit functions and differential equations, and denoting the set $\left(\epsilon_{1}, \cdots, \epsilon_{r}\right)$ by $\epsilon$, it is possible to prove

Lemma 2. For every system $H$ of variations the equations

$$
\varphi_{k}\left(x, y, y^{\prime}\right)=z_{k}(x)+\epsilon_{1} \zeta_{k}^{\prime}+\cdots+\epsilon_{r} \zeta_{k}^{r}
$$

determine a family of solutions $Y_{k}(x, \epsilon)$ with initial values

$$
Y_{k}\left(x_{1}, \epsilon\right)=y_{k}\left(x_{1}\right)+\epsilon_{1} \eta_{k}^{\prime}\left(x_{1}\right)+\cdots+\epsilon_{r} \eta_{k}^{r}\left(x_{1}\right),
$$

and of class $C^{\prime}$ in $\epsilon$ and $C^{\prime \prime}$ in $x$ near the values

$$
x_{1} \leqq x \leqq x_{2}, \quad \epsilon_{1}=\cdots=\epsilon_{r}=0
$$

for which it contains the arc $E$. The $\eta \zeta$-variations of the family with respect to the parameter $\epsilon_{\sigma}$ are $\eta_{k}^{\sigma}, \zeta_{k}^{\sigma}$.

For there is a neighborhood of the values $(x, y, \epsilon)$ satisfying the conditions

$$
(x, y) \text { on } E, \quad \epsilon_{1}=\epsilon_{2}=\cdots=\epsilon_{r}=0,
$$

in which the equations (7) have a set of solutions

$$
y_{k}^{\prime}=\psi_{k}(x, y, \epsilon)
$$

of class $C^{\prime},{ }^{*}$ and if to these equations are adjoined those of the set $d \epsilon_{\sigma} / d x=0$, then the existence theorems for differential equations establish the existence of a set of functions $Y_{k}(x, \epsilon)$ with the properties described in the lemma. $\dagger$ Since the $\eta \zeta$-variations of the family with respect to the parameter $\epsilon_{\sigma}$ satisfy the equation (6), since from (8) the initial values of the $\eta$ 's are the values $\eta_{k}^{\sigma}\left(x_{1}\right)$, and finally since the corresponding $\zeta$-variations are clearly the functions $\zeta_{k}^{\sigma}$,

${ }^{*}$ Bolza, Vorlesungen über Variationsrechnung, p. 166, § 22). See also Bliss, Fundamental Existence Theorems, The Princeton Colloquium Lectures, p. 19.

† Bolza, loc. cit., p. 168, \$\& 23, 24. 
it follows from Lemma 1 that the $\eta \zeta$-variations of the family with respect to $\epsilon_{\sigma}$ are the functions $\eta_{k}^{\sigma}, \zeta_{k}^{\sigma}$ as stated.

\section{The Lagrange multiplier rule}

In order to prove finally the result stated in the introduction two further lemmas will be useful.

Lemma 3. If a system $H$ of variations exists such that the determinant $\left|F_{\rho}\left(\xi^{\sigma}, \eta^{\sigma}\right)\right|$ is different from zero, then $E$ can not make $f_{1}$ a minimum.

For suppose such a system $H$ of variations $\xi_{1}^{\sigma}, \xi_{2}^{\sigma}, \eta_{k}^{\sigma}, \zeta_{k}^{\sigma}$ and consider the functions $Y_{k}(x, \epsilon)$ which they define as described in Lemma 2. If

$$
\begin{gathered}
X_{1}=x_{1}+\epsilon_{1} \xi_{1}^{\prime}+\cdots+\epsilon_{r} \xi_{1}^{r}, \quad X_{2}=x_{2}+\epsilon_{1} \xi_{2}^{\prime}+\cdots+\epsilon_{r} \xi_{2}^{r}, \\
f_{\rho}\left(\epsilon_{1}, \cdots, \epsilon_{r}\right)=f_{\rho}\left(X_{1}, Y\left(X_{1}, \epsilon\right), X_{2}, Y\left(X_{2}, \epsilon\right)\right)
\end{gathered}
$$

it is readily seen that for $\epsilon_{1}=\cdots=\epsilon_{r}=0$ the functional determinant $\left|\partial f_{\rho} / \partial \epsilon_{\sigma}\right|$ is identical with $\left|F_{\rho}\left(\xi^{\sigma}, \eta^{\sigma}\right)\right|$ and is different from zero. Hence the equations

$$
f_{1}-u=f_{2}=\cdots=f_{r}=0
$$

have an initial solution $u=f_{1}(0,0, \cdots, 0), \epsilon_{1}=\epsilon_{2}=\cdots=\epsilon_{r}=0$, at which their functional determinant with respect to $\epsilon_{1}, \cdots, \epsilon_{r}$ is different from zero. They have therefore solutions for $\epsilon_{1}, \cdots, \epsilon_{r}$ in terms of $u$, showing that there exist arcs in every neighborhood of $E$ satisfying

$$
\varphi_{\mu}=f_{2}=\cdots=f_{r}=0
$$

and giving $f_{1}$ values $u$ both greater and less than the value $f_{1}(0, \ldots, 0)$ which belongs to $E$. This proves the lemma.

Lemma 4. If $E$ makes $f_{1}$ a minimum there must be constants $l_{\sigma}$ not all zero such that the sum

$$
l_{1} F_{1}(\xi, \eta)+\cdots+l_{r} F_{r}(\xi, \eta)
$$

vanishes for every set of admissible variations satisfying equations (6).

By Lemma 3 the determinant $\left|F_{\rho}\left(\xi^{\sigma}, \eta^{\sigma}\right)\right|$ must vanish for every system $H$. Let $s$ be the maximum rank possible for the determinant and suppose $H$ selected so that this rank is actually attained. If the constants $l_{\sigma}$ are so chosen that the expression (9) vanishes for every variation in $H$ then (9) must also vanish for every admissible variation whatsoever. Otherwise the maximum rank of the determinant would be greater than $s$.

To prove the result described in the introduction let

$$
f=l_{1} f_{1}+\cdots+l_{r} f_{r},
$$




$$
\begin{aligned}
p_{1} & =\frac{\partial f}{\partial x_{1}}+\sum_{k} \frac{\partial f}{\partial y_{k 1}} y_{k 1}^{\prime}, & -p_{2} & =\frac{\partial f}{\partial x_{2}}+\sum_{k} \frac{\partial f}{\partial y_{k 2}} y_{k 2}^{\prime}, \\
q_{k 1} & =\frac{\partial f}{\partial y_{k 1}}, & -q_{k 2} & =\frac{\partial f}{\partial y_{k 2}},
\end{aligned}
$$

the end values of the variables in the expressions on the right being always those belonging to $E$. The values of $p_{1}, p_{2}, q_{k 1}, q_{k 2}$ can not all be zero since in that case the matrix in Hypothesis 4 of Section 1 would necessarily be of rank less than $r$. From equation (5), Lemma 4, and equation (6) it follows that the equations

$$
\begin{aligned}
& p_{1} \xi_{1}+\sum_{k} q_{k 1} \eta_{k 1}-p_{2} \xi_{2}-\sum_{k} q_{y_{k^{\prime}}} \eta_{k 2}=0 \\
& \int_{x_{1}}^{x_{2}} \sum_{k} \lambda_{k} \zeta_{k} d x=\int_{x_{1}}^{x_{2}} \sum_{k}\left(\Omega_{y_{k}} \eta_{k}+\Omega_{y_{k^{\prime}}} \eta_{k}^{\prime}\right) d x \\
&=\int_{x_{1}}^{x_{2}} \sum_{k}\left(\Omega_{y_{k}}-\frac{d}{d x} \Omega_{y_{k^{\prime}}}\right) d x+\left.\sum_{k} \Omega_{y_{k^{\prime}}} \eta_{k}\right|_{x_{1}} ^{x_{2}}
\end{aligned}
$$

hold for every set of admissible variations and every set of functions $\lambda_{k}(x)$ of class $C^{\prime}$ on $x_{1} x_{2}$. But the $\lambda$ 's can be chosen satisfying the $n$ differential equations with initial conditions

$$
\Omega_{y_{k}}-\frac{d}{d x} \Omega_{y_{k^{\prime}}}=0, \quad \Omega_{y_{k^{\prime}}}\left(x_{2}\right)=q_{k 2} .
$$

The sum of (11) and (12) then gives

$$
\int_{x_{1}}^{x_{2}} \sum_{k} \lambda_{k} \zeta_{k} d x=p_{1} \xi_{1}+\sum_{k}\left[q_{k 1}-\Omega_{y_{k^{\prime}}}\left(x_{1}\right)\right] \eta_{k 1}-p_{2} \xi_{2}
$$

as an equation which must hold for all admissible variations, and therefore by Lemma 1 for arbitrarily selected values $\xi_{1}, \xi_{2}, \eta_{k 1}, \zeta_{k}$. It follows readily then with the help of the Fundamental Lemma of the calculus of variations* and the initial conditions (13) for the functions $\lambda_{k}$ that

$$
\lambda_{\tau}=p_{1}=q_{k 1}-\Omega_{y_{k^{\prime}}}\left(x_{1}\right)=-p_{2}=q_{k 2}-\Omega_{y_{k^{\prime}}}\left(x_{2}\right)=0 \text {. }
$$

It is clear therefore that the last $n-m$ functions $\lambda_{r}$ vanish identically. But the first $m$ do not, since the last equations would otherwise make all of the constants $p_{1}, p_{2}, q_{k 1}, q_{k 2}$ vanish. The last four of equations (14) however imply that all determinants of order $r$ of the matrix

$$
\left\|\begin{array}{cccc}
\frac{\partial f_{\rho}}{\partial x_{1}}+\sum_{k} \frac{\partial f_{\rho}}{\partial y_{k 1}} y_{k_{1}}^{\prime} & \frac{\partial f_{\rho}}{\partial y_{k 1}} & \frac{\partial f_{\rho}}{\partial x_{2}}+\sum_{k} \frac{\partial f_{\rho}}{\partial y_{k 2}} y_{k_{2}}^{\prime} & \frac{\partial f_{\rho}}{\partial y_{k 2}} \\
0 & \Omega_{y_{k^{\prime}}}\left(x_{1}\right) & 0 & -\Omega_{y_{k^{\prime}}}\left(x_{2}\right)
\end{array}\right\|
$$

* Bolza, loc. cit., p. 25. 
vanish since they are obtained by multiplying the columns of this matrix by $l_{1}, \cdots, l_{r},-1$, adding, and equating the results to zero. The matrix (3) is obtained from the one just given by subtracting from the first and $(n+2) \mathrm{d}$ columns linear combinations of the others, and by introducing in the last row the terms $\Omega\left(x_{1}\right), \Omega\left(x_{2}\right)$ which are zero. This last step is entirely artificial and unnecessary, but it turns out that the matrix (3) gives more directly the customary results for many special cases. Since the matrix (3) is obtainable as just described from the last one, it follows that (3) also must have rank less than $r+1$.

The statement just proved that when $E$ minimizes $f_{1}$ there must exist $m$ functions $\lambda_{1}, \cdots, \lambda_{m}$ of class $C^{\prime}$ not all identically zero on $x_{1} x_{2}$, satisfying the equations (2), and making all determinants of order $r+1$ of the matrix (3) vanish, is equivalent to the statement that there must exist $m$ functions $\lambda$ of class $C^{\prime}$ satisfying the equations (2), and $r$ constants $l_{1}, \cdots, l_{r}$ not all zero satisfying with them the equations

$$
p_{1}=q_{k 1}-\Omega_{y_{k^{\prime}}}\left(x_{1}\right)=p_{2}=q_{k 2}-\Omega_{y_{k^{\prime}}}\left(x_{2}\right)=0,
$$

where the values of $p_{1}, q_{k 1}, p_{2}, q_{k 2}$ are defined by the equations (10).

For the equations (16) are the linear equations in $l_{1}, \cdots, l_{r},-1$ whose coefficients are the columns of the matrix (15). It is further a consequence of the existence theorems for differential equations that the only solutions of the equations (2) which can vanish simultaneously are the solutions

$$
\lambda_{1}=\cdots=\lambda_{n}=0 .
$$

Hence a set of solutions not identically zero must have values not all zero at $x_{1}$ and $x_{2}$, and consequently defines derivatives $\Omega_{y_{k^{\prime}}}\left(x_{1}\right), \Omega_{y_{k^{\prime}}}\left(x_{2}\right)$ not all zero. From the fact that the matrix (15) is of rank less than $r+1$ it follows readily therefore that multipliers $l_{1}, \cdots, l_{r}$ not all zero exist satisfying equations (16). Hence the first statement of the theorem implies the second. The proof that the second implies the first is immediate.

It is important to note that a minimizing arc $E$ is said to be normal for the problem under consideration when a system $H$ of variations can be so selected that the matrix $\left\|F_{\rho}\left(\xi^{\sigma}, \eta^{\sigma}\right)\right\|(\rho=2, \cdots, r: \sigma=1, \cdots, r)$ has rank $r-1$; otherwise it is abnormal.

For a normal minimizing arc $E$ the constant $l_{1}$ is always different from zero, and the constants and functions $l_{1}=1, l_{2}, \cdots, l_{r}, \lambda_{1}, \cdots, \lambda_{m}$ satisfying equations (2) and (16) are unique. In the abnormal case there always exists a set $l_{1}, \cdots, l_{r}, \lambda_{1}, \cdots, \lambda_{m}$ with $l_{1}=0$.

It is clear that $l_{1}$ can not be zero in the normal case since $l_{1}, \cdots, l_{r}$ make the expression (9) vanish for every set of admissible variations, and since the system $H$ can be selected so that the matrix 


$$
\left\|F_{\rho}\left(\xi^{\sigma}, \eta^{\sigma}\right)\right\|(\rho=2, \cdots, r: \sigma=1, \cdots, r)
$$

has rank $r-1$. The constants $l_{1}=1, l_{2}, \ldots, l_{r}$ are for a similar reason seen to be unique. Furthermore two systems $\lambda, \bar{\lambda}$ satisfying equations (2) and (16) with these constants would satisfy

$$
\Omega_{y_{k^{\prime}}}\left(x_{1}\right)=\bar{\Omega}_{y_{k^{\prime}}}\left(x_{1}\right) .
$$

Hence the differences of the corresponding elements of $\lambda$ and $\bar{\lambda}$ would vanish at $x_{1}$ and satisfy equations (2). They would therefore be identically zero.

The statement of the theorem with regard to the abnormal case is an immediate consequence of the way in which the constants $l_{1}, \ldots, l_{r}$ were originally determined.

\section{AN AUXILIARY THEOREM}

It was stated in Section 2 that it is always possible to adjoin to the functions $\varphi_{\mu}\left(x, y, y^{\prime}\right)$ a set of functions $\varphi_{\tau}\left(x, y, y^{\prime}\right)$ in such a way that the determinant $\left|\partial \varphi_{i} / \partial y_{k}^{\prime}\right|$ is different from zero along the arc $E$. The functions $\varphi_{\tau}$ can in fact be taken in the form

$$
\varphi_{\tau}=a_{\tau 1}(x) y_{1}^{\prime}+\cdots+a_{\tau n}(x) y_{n}^{\prime},
$$

where the functions $a_{\tau_{k}}$ are polynomials in $x_{1}$ and $x_{2}$. If the derivative $\partial \varphi_{\mu} / \partial y_{k}^{\prime}$ formed for the arc $E$ is denoted by $a_{\mu_{k}}(x)$, this result is an immediate consequence of the following theorem:

AUXILIARY THEOREM. If a matrix $\left\|a_{\mu_{k}}\right\|$ of continuous functions $a_{\mu_{k}}(x)$ has rank $m$ at every point of the interval $x_{1} x_{2}$, then $n-m$ additional rows of polynomials $a_{\tau k}(x)$ can always be selected so that the determinant $\left|a_{i k}\right|$ is everywhere different from zero on $x_{1} x_{2}$.

The rank of the matrix $\left\|a_{\mu_{k}}\right\|$ is unchanged if one row is multiplied by a continuous function of $x$ and added to another, and a proof of the theorem for the matrix so altered implies its validity also for the original one. If the first row of $\left\|a_{\mu k}\right\|$ is multiplied by a suitable factor and added to the second, the latter can be made orthogonal to the first, and by repetitions of this process every row can be made orthogonal to every other.

Consider then the linear equations in $u_{1}, \cdots, u_{n}$ whose coefficients are the rows of an orthogonal matrix $\left\|a_{\mu_{k}}\right\|$. If $n-m$ properly selected $u$ 's are arbitrarily chosen in a neighborhood of $x_{1}$ as continuous functions of $x$ not vanishing simultaneously, the $m$ remaining $u$ 's will be uniquely determined by the equations, since one at least of the determinants of the matrix is different from zero at $x_{1}$. Let $\xi$ then be the upper bound of the values $x$ defining intervals $x_{1} x$ on which the equations have continuous solutions not vanishing 
simultaneously. There is a neighborhood of $\xi$ in which one of the determinants of the matrix remains different from zero, and $x$ can be selected in this neighborhood between $x_{1}$ and $\xi$. The equations have solutions $u$ as just described on the interval $x_{1} x$ and it is possible to extend the definitions of $n-m$ of them so that they are continuous from $x_{1}$ to $\xi+\delta$. and not simultaneously vanishing to the right of $x$. If the $n-m$ extended $u$ 's have been properly selected the remaining $m$ will be uniquely determined from $x$ to $\xi+\delta$ by the equations, showing that $\xi$ can not be the upper bound described unless it is at $x_{2}$. Furthermore in the latter case the same argument shows that there exist $n$ continuous solutions $u$ not vanishing simultaneously on the interval $x_{1} x_{2}$.

If the row of functions $u$ so determined is adjoined to the matrix $\left\|a_{\mu_{i}}\right\|$ the resulting matrix has rank $r+1$ at every point of $x_{1} x_{2}$. For at a point where this were not true there would exist $m$ solutions $\nu$ of the $n$ equations

$$
u_{k}=\nu_{1} a_{1 k}+\cdots+\nu_{m} a_{m k} .
$$

The sum of these equations multiplied by the elements of the $\mu$ th row of the matrix would show that $\nu_{\mu}$ must vanish, since the $\mu$ th row is orthogonal to the $u$-row and all the others. The vanishing of all the $\nu$ 's would, however, contradict the fact that the $u$ 's do not vanish simultaneously.

It is clear therefore that one row of continuous functions $a_{m+1, k}$ can be adjoined to the matrix $\left\|a_{\mu_{k}}\right\|$ in such a way that the new matrix has rank $m+1$. Continuation of this process leads to a determinant $\left|a_{i k}\right|$ which is nowhere zero on $x_{1} x_{2}$. Furthermore since every continuous function can be approximated by a polynomial with any desired degree of accuracy it follows readily that the elements of the adjoined $n-m$ rows could be chosen as polynomials if desired.

\section{RELATIONS BETWEen DIFFERENT TYPES OF PROBLEMS}

For the problem of Bolza in non-parametric form the function to be minimized is

$$
U=\int_{x_{1}}^{x_{2}} f\left(x, y, y^{\prime}\right) d x+G\left(x_{1}, y\left(x_{1}\right), x_{2}, y\left(x_{2}\right)\right),
$$

while the equations of condition and end conditions are

$$
\begin{aligned}
\varphi_{a}\left(x, y, y^{\prime}\right) & =0 & & (\alpha=1, \cdots, p), \\
\psi_{\beta}(x, y) & =0 & & (\beta=1, \cdots, q), \\
\chi_{\gamma}\left(x_{1}, y\left(x_{1}\right), x_{2}, y\left(x_{2}\right)\right) & =0 & & (\gamma=1, \cdots, r) .
\end{aligned}
$$


He assumes that the determinant

$$
\left\|\begin{array}{lll}
\frac{\partial \varphi_{a}}{\partial y_{1}} & \ldots & \frac{\partial \varphi_{a}}{\partial y_{m}} \\
\frac{\partial \psi_{\beta}}{\partial y_{1}} & \cdots & \frac{\partial \psi_{\beta}}{\partial y_{m}}
\end{array}\right\| \quad \begin{aligned}
& (\alpha=1, \cdots, p) \\
& (\beta=1, \cdots, q) \\
& (m=p+q)
\end{aligned}
$$

is different from zero and that the end conditions are " reduced and nonsingular on $E$." The last means that the matrix of first derivatives

$$
\psi_{\beta}\left(x_{1}, y\left(x_{1}\right)\right), \quad \psi_{\beta}\left(x_{2}, y\left(x_{2}\right)\right), \quad \chi_{\gamma}\left(x_{1}, y\left(x_{1}\right), x_{2} y\left(x_{2}\right)\right)
$$

with respect to their arguments has rank $2 q+r$ at the values of the arguments on $E$.

When $f \equiv 0$ and the equations $\psi_{\beta}=0$ are absent this is exactly the form treated in the present paper, and the results which Bolza states in his Satz II* are exactly those of the next to last theorem of Section 2 above. On the other hand the problem just given is precisely that of determining an arc

$$
y_{0}(x), y_{1}(x), \cdots, y_{n}(x) \quad\left(x_{1} \leqq x \leqq x_{2}\right),
$$

which satisfies the differential equations

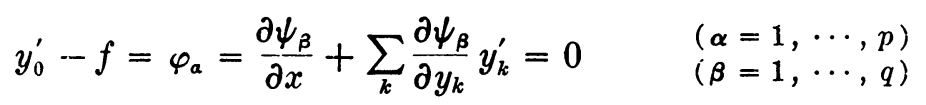

and gives the first of the functions

$$
\begin{array}{rr}
y_{02}-y_{01}+G\left(x_{1}, y\left(x_{1}\right), x_{2}, y\left(x_{2}\right)\right), \quad \psi_{\beta}\left(x_{1}, y\left(x_{1}\right)\right) & \\
\chi_{\gamma}\left(x_{1}, y\left(x_{1}\right), x_{2}, y\left(x_{2}\right)\right) & (\beta=1, \cdots, q) \\
& (\gamma=1, \cdots, \gamma)
\end{array}
$$

a minimum value while making the rest vanish. It is readily seen that this has the form treated in the preceding sections, and by applying the results of Section 2 it turns out that the various constants and functions for the two problems correspond as in the following table:

(A) $l_{1}, l_{1+\beta}-\lambda_{p+\beta}\left(x_{1}\right), \quad \lambda_{p+\beta}\left(x_{2}\right), l_{1+\beta}, l_{1+q+\gamma}, \lambda_{a}(x),-\lambda_{p+\beta}^{\prime}(x)$

(B) $l_{0}, \quad l_{\beta}^{\prime \prime}, \quad l_{\beta}^{\prime}, \quad l_{\beta}, l_{q+\gamma}, \lambda_{a}(x), \quad \mu_{\beta}(x)$

$$
\alpha=1, \cdots, p ; \quad \beta=1, \cdots, q ; \quad \gamma=1, \cdots, r .
$$

The second row is the on: containing the elements in Bolza's notation which appear in his paper. From this table and the next to last theorem of Section 2 one deduces at once bolza's Satz II.

University of Chica(io

September, 1917

* Mathematische Annalen, loc. cit., p. 438. 